\title{
QUANTUM IMPLICATIONS OF RAY SPLITTING
}

\author{
R. BLÜMEL \\ Fakultät für Physik, Albert-Ludwigs-Universität \\ Hermann-Herder-Str. 3, 79104 Freiburg, Germany
}

\begin{abstract}
Ray splitting is a universal phenomenon that occurs in all wave systems with sharp interfaces. Quantum implications of ray splitting are: (i) the importance of non-Newtonian orbits for the density of states in the semiclassical limit, (ii) ray-splitting corrections to the average density of states and (iii) the need to include non-Newtonian orbits in trace formulas for the oscillating part of the density of states. The signatures of non-Newtonian orbits in the density of states have recently been identified experimentally ( $\mathrm{L}$. Sirko, P.M. Koch, R. Blümel, Phys. Rev. Lett. 78, 2940 (1997)).
\end{abstract}

PACS numbers: $05.45 .+\mathrm{b}$

In 1948 Feynman introduced a particularly illuminating representation of quantum mechanics [1]. According to Feynman the transition amplitude of a particle from point $P$ to point $Q$ in the phase space is given by a sum of complex phases computed on the basis of all possible phase-space paths connecting $P$ with $Q$. In order to obtain the exact quantum transition amplitude all paths in $\mathcal{S}$ are equally important. It is, however, possible to bring out particular subclasses of $\mathcal{S}$ by a judicious choice of quantum problems. Let us focus on the class of potential problems. For such problems it is convenient to divide $\mathcal{S}$ into the two disjoint subsets of Newtonian $(\mathcal{N})$ and non-Newtonian $(\overline{\mathcal{N}})$ trajectories. The Newtonian trajectories are the solutions of the classical canonical equations. For smooth potentials and sufficiently small $\hbar$ very good approximations to the quantum transition amplitudes can be obtained on the basis of $\mathcal{N}$ alone. The contribution of the rest of the paths is near zero because of destructive interference. In the case of non-smooth potentials particular subclasses of $\overline{\mathcal{N}}$ have to be kept besides the trajectories contained in $\mathcal{N}$ for a good representation of transition amplitudes. In other words, potentials with steps and other types of irregularities may be used as "projectors" to bring out the effects of particular classes of non-Newtonian trajectories. In the case of step potentials the importance of the non-Newtonian orbits survives the $\hbar \rightarrow 0$ limit. Thus Newtonian mechanics is not the only mechanics important in the semiclassical limit of quantum mechanics. In the case of step potentials, e.g., the underlying orbit structure in the semiclassical limit is obtained from a nondeterministic, non-Newtonian mechanics [2-6]. 
In order to illuminate the new concept of a non-Newtonian mechanics consider the following potential:

$$
V(x, w)= \begin{cases}0, & \text { for } x \leq 0 \\ V_{0} x / w, & \text { for } 0 \leq x \leq w \\ V_{0}, & \text { for } x \geq w .\end{cases}
$$

For $w \rightarrow 0$ we obtain a step potential. We are particularly interested in the scattering of waves off the potential (1) for $E>V_{0}$. In the asymptotic region to the left of the potential the wave function is given by

$$
\psi(x)=\mathrm{e}^{\mathrm{i} k x}+r \mathrm{e}^{-\mathrm{i} k x}, \quad x \leq 0, \quad k=\sqrt{2 m E / \hbar^{2}} .
$$

To the right of the potential we have

$$
\psi(x)=t \mathrm{e}^{\mathrm{i} k x}, \quad x \geq w, \quad \kappa=\sqrt{2 m\left(E-V_{0}\right) / \hbar^{2}} .
$$

Since (1) is a piecewise linear potential, $r$ can be computed with the help of Airy functions. We obtain

$$
r=\frac{\mathrm{i} k \kappa C_{1}+k q C_{2}-\kappa q C_{3}-\mathrm{i} q^{2} C_{4}}{\mathrm{i} k \kappa C_{1}+k q C_{2}+\kappa q C_{3}+\mathrm{i} q^{2} C_{4}},
$$

where

$$
q=\left(\frac{2 m V_{0}}{\hbar^{2} w}\right)^{1 / 3} .
$$

The constants $C$ are given by

$$
\begin{aligned}
& C_{1}=\mathrm{Ai}(-\beta) \mathrm{Bi}(-\alpha)-\mathrm{Ai}(-\alpha) \mathrm{Bi}(-\beta), \\
& C_{2}=\mathrm{Ai}(-\alpha) \mathrm{Bi}^{\prime}(-\beta)-\mathrm{Ai}^{\prime}(-\beta) \mathrm{Bi}(-\alpha), \\
& C_{3}=\operatorname{Ai}(-\beta) \mathrm{Bi}^{\prime}(-\alpha)-\mathrm{Ai}^{\prime}(-\alpha) \operatorname{Bi}(-\beta), \\
& C_{4}=\mathrm{Ai}^{\prime}(-\beta) \mathrm{Bi}^{\prime}(-\alpha)-\mathrm{Ai}^{\prime}(-\alpha) \mathrm{Bi}^{\prime}(-\beta),
\end{aligned}
$$

where

$$
\alpha=q w E / V_{0}, \quad \beta=q w\left(\frac{E}{V_{0}}-1\right) .
$$

We are mainly interested in the double limit $w \rightarrow 0, \hbar \rightarrow 0$. Investigation of Eq. (4) shows that the two limits do not commute. Moreover, let $w$ and $\hbar$ approach 0 , but keep their ratio $\nu=\hbar / w$ constant. Then it is easy to show that for $\hbar \rightarrow 0$ the reflection amplitude is $r \neq 0$ and depends on $\nu$. A finite $r$ for $\hbar \rightarrow 0$ means that in the classical limit there exist trajectories reflecting off the step although $E>V_{0}$. These must clearly be non-Newtonian trajectories since Newtonian trajectories transmit with probability 1 for $E>V_{0}$. The probability for a particle to go left is $p=|r|^{2}$, the probability to go right is $1-p$. The decision about whether to reflect (go left) or to transmit (go right) is left to chance, governed by $p$. Thus, the non-Newtonian dynamics for a step potential is non-deterministic. If we represent the incoming path of the particle by a ray, there are two possibilities for this ray to leave the step: the particle may reflect (with probability $p$ ) or transmit (with 
probability $1-p$ ). Thus, one incident ray creates two (or sometimes more) outgoing riuys. We call this situation "ray splitting".

Ray splitting is a universal phenomenon that occurs in all wave systems with sharp interfaces in the limit of small wavelength. Examples are the splitting of light rays at the interface between two transparent dielectrics, the splitting of acoustic rays at the interface between two media of different density, the splitting of rays associated with water surface waves at the interface between two different depths, and finally the splitting of rays associated with de Broglie matter waves in quantum mechanics at the position of a potential step.

The wave implications of ray splitting were recently studied in the context of acoustic and quantum systems [2-7]. Major findings were the importance of non-Newtonian orbits for the oscillating part of the density of states [2-6], the necessity of correcting the Weyl formula [8] for the average density of states of ray-splitting systems [7] and the need to modify existing trace formulas [8] to include non-Newtonian periodic orbits [2]. Additional quantum implications are the existence of new classes of scars in the quantum wave functions [3].

The signatures of periodic non-Newtonian orbits were recently identified experimentally $[5,6]$ in the context of microwave resonance spectroscopy. We used thin dielectric- and metal-loaded cavities to generate ray-splitting of microwaves a: sharp air/teflon and air/metal interfaces. The Fourier transform of the measured density of resonances shows peaks at the optical path lengths of non-Newtonian orbits. Since for thin microwave resonators the electromagnetic Helmholtz equation and the quantum Schrödinger equation are equivalent $[9,10]$, these experiments are of direct relevance for quantum ray-splitting systems.

Having established the importance of non-Newtonian orbits experiments should now aim at testing the ray-splitting correction of the Weyl formula. In order to do this the experiments have to be improved in such a way that $\approx 500$ levels can be measured without missing a single one. Not missing a single level is a stringent experimental constraint which may be achieved with the help of numerical support. It was recently demonstrated [4] that a few hundred levels are indeed enough for a first qualitative test of the ray-splitting correction.

The theory of ray-splitting systems is also not complete yet. For example, the ray-splitting correction derived analytically in Ref. [7] applies only to rectilinear ray-splitting boundaries. What is missing is the computation of the correction for curved ray-splitting boundaries.

Another promising route for theoretical research is the identification of prebifurcation ghosts [11] in ray-splitting systems. As non-Newtonian orbits undergo much the same type of bifurcations as Newtonian orbits, we expect the existence of non-Newtonian ghosts in ray-splitting systems. Theoretical work on the identification of the sigilatures of non-Newtonian ghosts is currently in progress.

Fruitful discussion with Prof. Fritz Haake on the nature of ghost orbits are gratefully acknowledged. The author is grateful for financial support by the Deutsche Forschungsgemeinschaft. 


\section{References}

[1] R.P. Feynman, Rev. Mod. Phys. 20, 367 (1948).

[2] L. Couchman, E. Ott, T.M. Antonsen, Jr., Phys. Rev. A 46, 6193 (1992).

[3] R. Blümel, T.M. Antonsen, B. Georgeot, E. Ott, R.E. Prange, Phys. Rev. Lett. 76, 2476 (1996); Phys. Rev. E 53, 3284 (1996).

[4] A. Kohler, G.H.M. Killesreiter, R. Blümel, Phys. Rev. E, in press.

[5] L. Sirko, P.M. Koch, R. Blümel, Phys. Rev. Lett. 78, 2940 (1997).

[6] Sz. Bauch, A. Błędowski, L. Sirko, P.M. Koch, R. Blümel, submitted to Phys. Rev. E.

[7] R.E. Prange, E. Ott, T.M. Antonsen, Jr., B. Georgeot, R. Blümel, Phys. Rev. E 53, 207 (1996).

[8] M.C. Gutzwiller, Chaos in Classical and Quantum Mechanics, Springer, New York 1990.

[9] J.D. Jackson, Classical Electrodynamics, Wiley, New York 1975.

[10] H.-J. Stöckmann, J. Stein, Phys. Rev. Lett. 64, 2215 (1990).

[11] M. Kuś, F. Haake, D. Delande, Phys. Rev. Lett. 71, 2167 (1993). 